\title{
Corneoscleral transplantation for end stage corneal disease
}

\author{
Lawrence W Hirst, Graham A Lee
}

\begin{abstract}
Aim-To describe the prognosis and complications of corneoscleral transplantation in the management of end stage eye disease.

Methods-A case series is presented of 23 patients who have undergone corneoscleral transplantation ( $\geqslant 11 \mathrm{~mm})$. Patients were examined for visual acuity, intraocular pressure, recurrence of disease process, epithelialisation of the graft, signs of rejection, and other potential complications.

Results-14 patients retained their eye, with six maintaining a clear graft. Vision ranged from $6 / 30$ to no perception of light. 13 patients developed glaucoma (range 25-69 $\mathrm{mm} \mathrm{Hg}$ ), with six patients requiring surgical intervention. 12 patients required tarsorrhaphy to promote epithelialisation. Only two grafts resulted in typical rejection.

Conclusions-The technique of corneoscleral transplantation can salvage otherwise end stage eye disease, but the results are poor with respect to maintenance of vision. These patients need careful follow up because of potential complications of glaucoma, epithelial defects, rejection, and recurrence of disease.
\end{abstract}

(Br F Ophthalmol 1998;82:1276-1279)

Correspondence to: Professor Lawrence W Hirst, 2nd Floor Lions Building,

Princess Alexandra Hospital, Ipswich Road,

Woolloongabba, Queensland, Australia, 4102 .

Accepted for publication 21 April 1998 End stage corneal disease due to conditions such as extensive infection and epithelial downgrowth, have been treated in the past by anterior segment reconstruction using large corneoscleral grafts. The results have been very variable. ${ }^{1-6}$ We present a series of 23 consecutive patients requiring anterior segment reconstruction using corneoscleral grafts $\geqslant 11 \mathrm{~mm}$ in diameter.

\section{Materials and methods}

Patients with end stage corneal infections, widespread coagulative necrosis of the cornea, or epithelial downgrowth were treated by one surgeon (LWH). In all of the patients, the surgery was performed as a procedure designed to save the eye from certain loss as a result of the disease process.

Twenty three consecutive patients were treated over a 10 year period with corneoscleral grafts larger than or equal to $11 \mathrm{~mm}$ in diameter. Two grafts were $11 \mathrm{~mm}, 10$ grafts were $12 \mathrm{~mm}$, seven grafts were $13 \mathrm{~mm}$, and four grafts were $14 \mathrm{~mm}$ (Table 1). Eight of the patients were treated for bacterial keratitis with or without endophthalmitis, seven for fungal keratitis with or without endophthalmitis, three for acanthamoeba keratitis, four for epithelial downgrowth, and one for a rheumatoid corneal perforation.

Common features of the surgical technique included a total limbal peritomy in an endeavour to save possible limbal stem cells at the conjunctival edge and identification and fixation of the four recti muscles. Oversized trephines of 11-14 $\mathrm{mm}$ were used to mark the scleral surface and partially trephine through this tissue. Cautery was used to provide haemostasis and the anterior chamber was entered obliquely with a diamond knife leaving a ledge of sclera (Fig 1). The entire cornea was

Table 1 Patient characteristics, condition, graft size, outcome, and graft status

\begin{tabular}{|c|c|c|c|c|c|c|}
\hline No & Age & Sex & Condition & Size & Outcome & Graft status \\
\hline 1 & 73 & $M$ & Acanthamoeba & 13 & Count fingers & clear \\
\hline 2 & 72 & $\mathrm{~F}$ & Fungal & 12 & Count fingers & clear \\
\hline 3 & 52 & $M$ & Fungal & 12 & Enucleation & \\
\hline 4 & 62 & $M$ & Bacterial & 12 & Hand movement & opaque \\
\hline 5 & 71 & $M$ & Bacterial & 12 & Enucleation & \\
\hline 6 & 72 & $\mathrm{~F}$ & Fungal & 12 & Light perception & opaque \\
\hline 7 & 76 & $\mathrm{~F}$ & Epithelial downgrowth & 13 & Light perception & opaque \\
\hline 8 & 81 & $\mathrm{~F}$ & Epithelial downgrowth & 13 & Enucleation & \\
\hline 9 & 77 & $\mathrm{~F}$ & Acanthamoeba & 12 & $6 / 120$ & clear \\
\hline 10 & 60 & $M$ & Bacterial & 13 & Enucleation & \\
\hline 11 & 67 & $M$ & Fungal & 13 & Enucleation & \\
\hline 12 & 65 & $\mathrm{~F}$ & Acanthamoeba & 12 & Light perception & clear \\
\hline 13 & 50 & $M$ & Epithelial downgrowth & 14 & Hand movement & opaque \\
\hline 14 & 76 & $M$ & Bacterial & 12 & $6 / 60$ & clear \\
\hline 15 & 65 & $M$ & Epithelial downgrowth & 12 & Enucleation & \\
\hline 16 & 65 & $\mathrm{~F}$ & Bacterial & 14 & Evisceration & \\
\hline 17 & 70 & $M$ & Bacterial & 14 & Enucleation & \\
\hline 18 & 21 & $M$ & Fungal & 11 & $3 / 120$ & opaque \\
\hline 19 & 73 & $\mathrm{~F}$ & Bacterial & 11 & No light perception & opaque \\
\hline 20 & 80 & $\mathrm{~F}$ & Bacterial & 14 & $6 / 30$ & clear \\
\hline 21 & 72 & $\mathrm{~F}$ & Rheumatoid & 12 & Hand movement & opaque \\
\hline 22 & 45 & $\mathrm{~F}$ & Fungal & 13 & Enucleation & \\
\hline 23 & 76 & $M$ & Fungal & 13 & Light perception & opaque \\
\hline
\end{tabular}

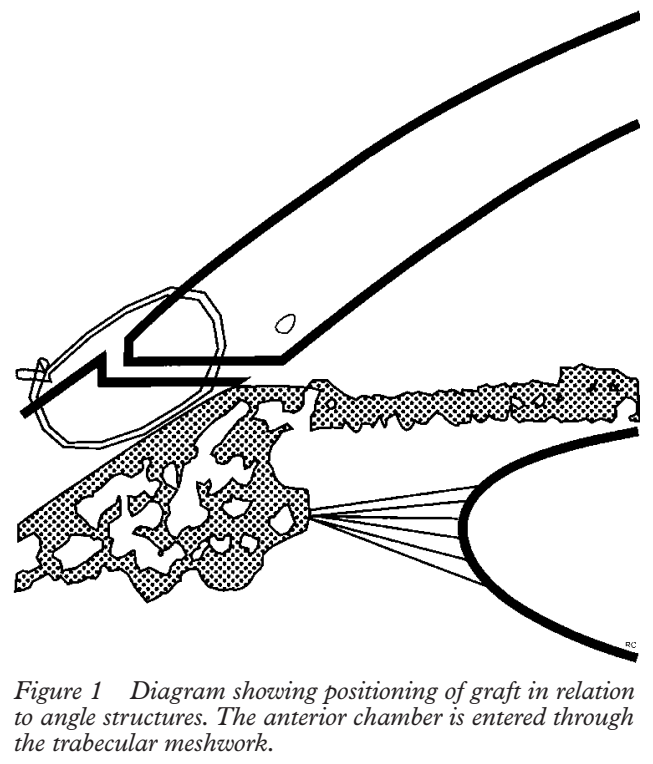



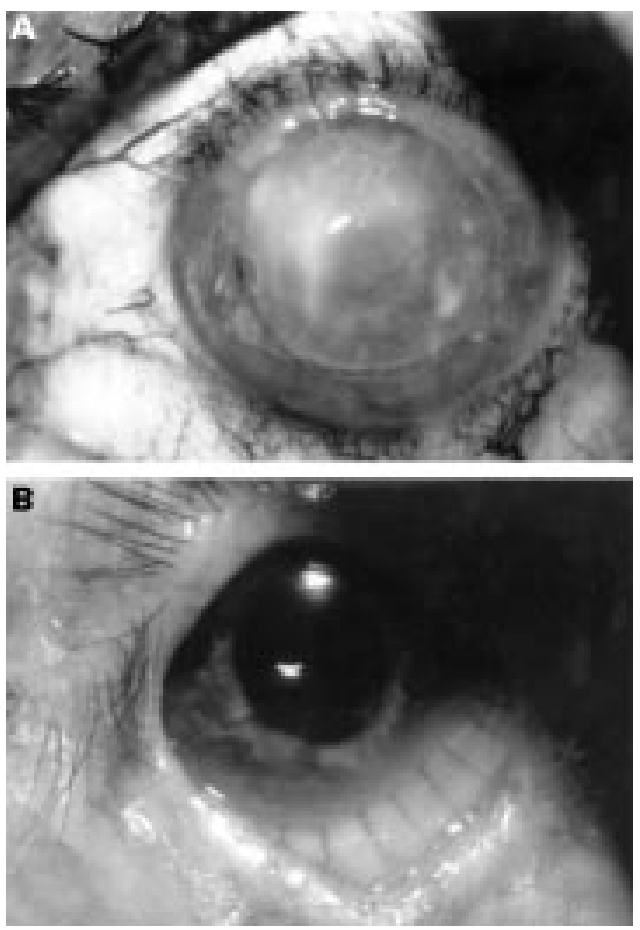

Figure 2 External photograph of patient with bacteria (Staphylococcus aureus) keratitis in a corneal graft $(A)$ and 2 years after a $12 \mathrm{~mm}$ corneoscleral graft (B).

and also for culture in appropriate cases. Where infective conditions or epithelial downgrowth involved the iris, it was removed and in six cases, the lens was removed. In these cases the posterior capsule was not preserved and a vitrectomy was performed. In no cases was the ciliary body resected. The donor tissue was trephined from a whole eye used within 24
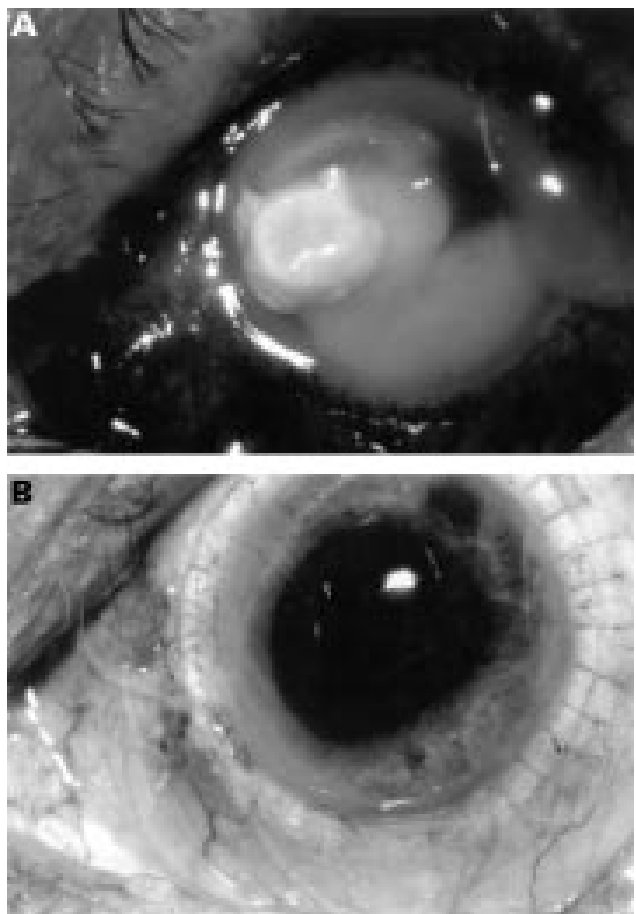

Figure 3 External photograph of patient with fungal keratitis $(A)$ and 7 months after a $12 \mathrm{~mm}$ corneoscleral graft, revealing the Molteno tube and 9/0 nylon sutures with exposed knots $(B)$.
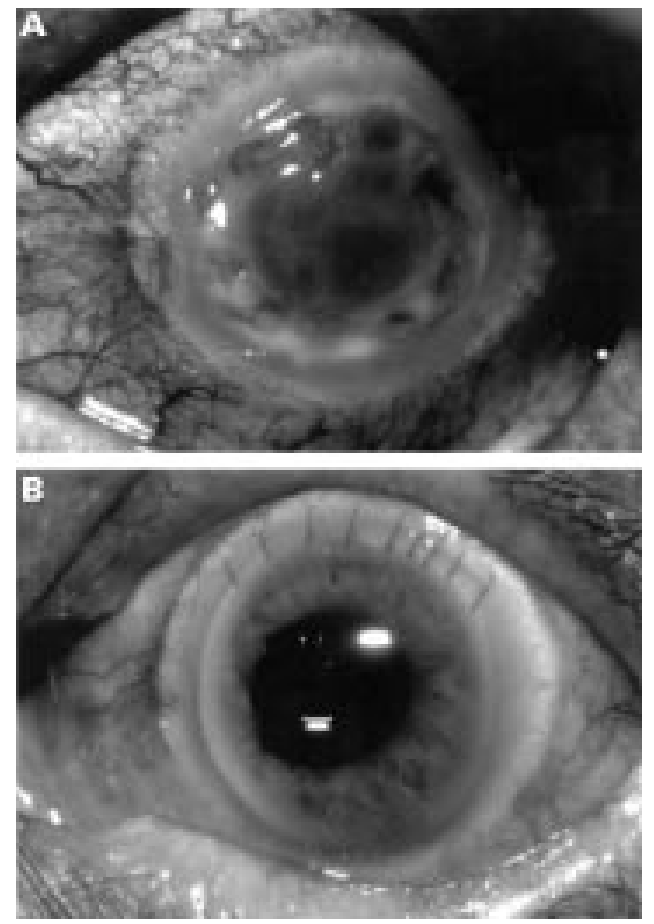

Figure 4 External photograph of patient with acanthamoeba keratitis demonstrating multiple corneal biopsy sites $(A)$ and 2.5 years after a $12 \mathrm{~mm}$ corneoscleral graft $(B)$.

hours of death and stored in a moist chamber using a trephine of the same size as that used on the recipient. Viscoelastic material was placed on the recipient bed and the donor corneoscleral graft was sutured into place onto the scleral ledge using interrupted $10 / 0$ or $9 / 0$ nylon sutures. The knots were frequently unable to be buried. The conjunctiva was then closed to cover the peripheral part of the graft and intensive topical steroids together with intravenous antimicrobial therapy, where applicable, were continued. In three cases, partial, permanent lateral tarsorrhaphy was performed at the time of the anterior segment reconstruction or in nine cases at the first sign of persistent epithelial defect. All cases were performed under general anaesthesia with surgery taking an average of 2 hours. Postoperative steroid therapy was 2 hourly prednisolone acetate $1 \%$ for $3-4$ weeks and then a slow titration to one drop per day thereafter long term.

Subsequent glaucoma was treated with topical medications and oral acetazolamide (Diamox) or with the placement of a Molteno valve or application of cyclocryotherapy. Frequent follow up was maintained with daily visits for the first few days after surgery, followed by weekly visits for 1-2 months and then monthly visits thereafter.

Corneal graft rejections were treated vigorously with topical half hourly prednisolone acetate $1 \%$ for 1 day, then hourly for 2 days and slow titration depending on clinical response and toxicity. Some cases required the addition of oral prednisone $60 \mathrm{mg}$ per day and/or subconjunctival dexamethasone $5 \mathrm{mg}$. 


\section{Results}

In nine patients, enucleation or evisceration were required, primarily because of recurrence of the underlying disease process. The mean time between corneoscleral grafting and removal of the eye was 17 months, but ranged from 0 to 80 months. In one of these patients, an expulsive haemorrhage occurred during surgery requiring immediate evisceration. In the remaining 14 patients, visual acuity ranged from no light perception (one), light perception (four), hand movement (three), counting fingers (two), 3/120 (one), 6/120 (one), 6/60 (one), and 6/30 (one) (Table 1).

Twelve of the patients required tarsorrhaphy to promote epithelialisation of the cornea within the first 6 weeks after the anterior segment surgery. In three patients prophylactic tarsorrhaphy was performed. The epithelial defects were uniformly present in the interpalpebral zone and associated with early stromal thinning. These were recalcitrant to treatments including tissue adhesive, vitamin A ointment, and contact lens, but responded well to tarsorrhaphy. In one patient, a further transplant was required for a perforation occurring in the corneoscleral graft under an area of persistent epithelial defect. Three patients in the tarsorrhaphy group ultimately required enucleation.

Thirteen patients developed glaucoma (range 25-69 $\mathrm{mm} \mathrm{Hg}$ ), with seven of the patients responding satisfactorily to topical and oral medication. Six patients required surgical intervention for glaucoma, four with a Molteno valve and two with repeated cyclocryotherapy. One of these patients having repeat cyclocryotherapy required enucleation due to hypotony, but this patient also had a recurrence of epithelial downgrowth. Four patients developed retinal detachment, with two patients requiring enucleation and the other two eyes resulting in vision less than hand movements.

Two patients could be defined as having typical rejection episodes with the corneas becoming opaque, while five corneas slowly failed without definite reason. These may represent atypical graft rejection or endothelial failure. In one other patient, the cornea became opaque after the use of intracameral amphotericin. In total, six out of 13 retained eyes maintained clear grafts with a follow up of 837 days (SD 912 days) with a range of 49-2701 days (Figs 2-4).

\section{Discussion}

Large corneoscleral grafts have been undertaken by a number of surgeons with the largest series first reported in $1973 .{ }^{1}$ In this series in Malawi, 14 patients underwent total keratoplasty with disappointing results after the primary procedure and some recovery of vision in patients with further corneal transplants or keratoprostheses. There appeared to be a uniform opacification of most grafts within the first 12 months of surgery with only three grafts remaining clear for more than 18 months. In 1989, Kirkness et al reported on 17 grafts larger than $9.5 \mathrm{~mm}$ with a minimum follow up of 6 months. ${ }^{2}$ The principles of the sur- gical removal were clearly described and were similar to those used in this series. Vision better than $6 / 18$ was obtained in over $40 \%$ of the cases, while vision was less than $6 / 60$ in about $30 \%$. In none of the cases was enucleation required and in none did endophthalmitis occur or corneal infection recur. Corneal graft rejections were seen in eight of the patients with three of the grafts failing as a result. Interestingly, no comment was made on prolonged epithelial problems in this series of large grafts. Various explanations were given as to the reasons for the relatively low incidence of glaucoma and rejection.

In 1992, Cobo et al described the use of 14 $\mathrm{mm}$ sclerokeratoplasty in five eyes for a variety of diseases. ${ }^{3}$ Attempted preservation of angle structures by bevelling into clear cornea both in the donor and the recipient was undertaken in a manner similar to our technique. Both interrupted, horizontal, running sutures, and the use of angle support sutures were placed. A lateral tarsorrhaphy was performed routinely. In at least four of these six eyes, a further corneal transplant was required after loss of clarity of the initial graft. One patient required long term glaucoma therapy and most angles appeared to be open as examined by gonioscopy.

In 1992, Naumann and Rummelt reported on the treatment of cystic and diffuse epithelial ingrowth by the use of en bloc dissection including large corneoscleral grafts which were frequently eccentric and did not include the entire cornea. ${ }^{4}$ This series was noteworthy for the lack of recurrence of ingrowth and cysts and for the retention of useful vision. However, total corneoscleral replacement was not a routine part of this procedure.

In 1996, Redbrake and Buchal described the use of keratoplasty with a scleral rim in the treatment of severe chemical eye burns. ${ }^{6}$ Despite all nine eyes being saved, only two of the 12 grafts remained clear. Reasons for graft failure were multifactorial; however, the majority were thought to be due to epithelial problems. Multiple other reports of individual cases of total penetrating keratoplasty have been reported. ${ }^{5-14}$

Our series reflects a high rate of enucleation, principally as a result of recurrent primary disease, usually either infection or recurrence of epithelial downgrowth. This occurred despite the aggressive use of both intraocular and intravenous antibiotics after the initial anterior segment reconstruction. Among these were four patients with a previously described paecilomyces anterior segment infection which was particularly resistant to surgical and medical treatment. ${ }^{15}$

Three major problems expected in this patient group are recurrence of initial disease, glaucoma, and rejection. Recurrence of disease was by far the most common end point and reflected the fulminating nature of disease in this group of patients. These end stage conditions would have inevitably led to loss of the eye without any attempt at salvage surgery.

Glaucoma was a frequent complication, but could be controlled with topical medications or 
with a Molteno valve. Corneoscleral grafting would be expected to cause almost total angle destruction and hence result in absolute glaucoma. The possibilities of why absolute glaucoma does not result include the presence of cyclodialysis clefts, other routes of aqueous outflow, partial ciliary body disinsertion, and even that the transplanted trabecular meshwork may function. ${ }^{8}$ The use of angle preserving mattress sutures has been described together with the preservation of a ledge of sclera extending into the cornea from the original $12 \mathrm{~mm}$ or larger trephine site. ${ }^{3}$

Typical episodes of rejection were not common in those eyes which survived the first 2 or 3 weeks after corneoscleral grafting. This is despite several studies which indicate that there is increased rejection in larger grafts. ${ }^{16}{ }^{17}$ However, atypical rejection which could occur in such large grafts, may explain the common occurrence of progressive corneal oedema. A reduction of density of Langerhan's dendritic cells has been used to explain this phenomenon. $^{2}$

Of greatest difference between the current report and previous studies has been the recurrent and persistent nature of epithelial defects In one of the other series, immediate tarsorrhaphy was performed at the time of corneoscleral graft and as observed in three of our patients, this may prevent subsequent epithelial problems. ${ }^{3}$

It could be expected by removal of the entire cornea, particularly including the corneal limbus, that the patient's limbal stem cells would be totally destroyed. Ocular resurfacing of the cornea would then be possible only by the donor epithelium whose ultimate success relies on donor stem cells. Rejection of these allogenic stem cells is highly likely which would then result in conjunctival epithelialisation of the corneal surface from host tissue, with a vascularised, slightly opaque epithelium covering the corneal graft. In none of our cases was an actual epithelial rejection line seen and most of the epithelial defects occurred too early for this simple explanation. It is quite possible that total corneal denervation as a result of this procedure may play a part in the recurrent epithelial breakdown, but it is surprising that none of the other series have described this phenomenon. It is also interesting to note that prophy- lactic tarsorrhaphy appeared to prevent the breakdown of the corneal epithelium and that tarsorrhaphy after the occurrence of epithelial breakdown assisted in the rehabilitation of the ocular surface.

Large corneoscleral grafts may be required in an attempt to salvage an eye which would otherwise be lost; however, the results are poor relating to maintenance of vision. Although the complications of uncontrolled glaucoma and corneal graft rejection are uncommon, persistent epithelial defects are seen in virtually all patients and require tarsorrhaphy for healing to take place. This procedure can be recommended only for patients who have fulminating eye disease. They must be prepared for the likelihood of poor vision, the possibility of a tarsorrhaphy and the requirement for frequent follow up.

Supported by the Prevent Blindness Foundation. Presented in part at the American Academy of Ophthalmology Annual Meeting, San Francisco, California, October 1997.

We would like to thank Richard Campbell, B Art, Central Medical Illustration Unit, Royal Brisbane Hospital.

1 Ticho U, Ben-Sira I. Total keratoplasty. Arch Ophthalmol 1973;90:104-6.

2 Kirkness CM, Ficker LA, Rice NSC, et al. Large corneal grafts can be successful. Eye 1989;3:48-55.

3 Cobo M, Ortiz JR, Safran SG. Sclerokeratoplasty with maintenance of the angle. Am f Ophthalmol 1992;113:5337 .

4 Naumann GOH, Rummelt V. Block excision of cystic and diffuse epithelial ingrowth of the anterior chamber. Arch diffuse epithelial ingrowth of
Ophthalmol 1992;110:223-7.

5 Reynolds MG, Alfonso E. Treatment of infectious scleritis and keratoscleritis. Am f Ophthalmol 1991;112:543-7.

6 Redbrake C, Buchal V. Keratoplastik mit Skleraring nach schwersten Veratzungen des vorderen Augenabschnittes. Klin Monatsbl Augenheilkd 1996;208:145-51.

7 Mendez EA, Daza MT. Sclerokeratoplasty in a case of corneal keloid. Cornea 1991;10:183-4.

8 Lustbader JM, Barraquer J, Green WR. Corneoscleral autotransplant. Arch Ophthalmol 1988;106:953-7.

9 Mohammadi H, Morton RL, Wetterholm D. Total keratoplasty in peripheral corneal degeneration. Ophthalmic Surg 1982;13:400-2

10 Taylor DM, Stern AL. Reconstructive keratoplasty in the management of conditions leading to corneal destruction. Ophthalmology 1980;87:892-904.

11 Waring GO III, Beernink DH. Scleral ring as template for corneoscleral graft. Am f Ophthalmol 1978;85:258-60.

12 Barraquer J. Total penetrating keratoplasty. Proc R Soc Med 1961;54:1116-8.

13 Maumenee AE. Penetrating autokeratoplasty of the entire cornea. Am f Ophthalmol 1959;47:125-33.

14 Castroviejo R. Total penetrating keratoplasty. A preliminary report. Am f Ophthalmol 1951;34:1697-706.

15 Hirst LW, Sebban A, Whitby RM, et al. Non-traumatic mycotic keratitis. Eye 1992;6:391-5.

16 Cherry PMH, Pashby RC, Tadros ML, et al. An analysis of corneal transplantation: I Graft clarity. Ann Ophthalmol 1979;11:461-9.

17 Völker-Dieben HJ, Kok-Van Alphen CC, Lansbergen Q, et al. Different influences on corneal graft survival in 539 transplants. Acta Ophthalmol 1982;60:190-202. 